\title{
HAMILTON EQUATION ON ALMOST PSEUDO KÄHLER-WEYL MANIFOLDS FOR MECHANICAL SYSTEMS
}

\author{
Zeki Kasap \\ Department of Mathematics, Elementary Education, Faculty of Education, \\ Pamukkale University, Kinikli Campus, Denizli, Turkey
}

\begin{abstract}
This paper aims to present Hamiltonian formalism for mechanical systems using pseudoKähler Weyl manifolds, which represent an interesting multidisciplinary field of research. As a result of this study, partial differential equations will be obtained for movement of objects in space and solutions of these equations will be made by using the Maple computer program. In this study, some geometrical, relativistic, mechanical, and physical results related to pseudo-Kähler Weyl mechanical systems are also given.
\end{abstract}

Keywords: pseudo Kähler Weyl geometry, Hamiltonian systems, conformal, dynamical systems.

${ }^{*}$ Corresponding author.

E-mail address: zekikasap@hotmail.com (Zeki Kasap).

Copyright ( 2015 Scientific Advances Publishers

2010 Mathematics Subject Classification: 53A30, 34B20, 37K65, 70G60.

Submitted by Jianqiang Gao.

Received December 12, 2015 


\section{Introduction}

The linear distance between two points can be found easily by Riemann metric, which is very useful and is defined inner product. Many scientists have used the Riemann metric. Einstein was one of the first studies in this field. Einstein discovered which the Riemannian geometry and successfully used it to describe general relativity in the 1910 that is actually a classical theory for gravitation. However, the universe is really completely not like Riemannian geometry. Each path between two points is not always linear. Also, orbits of move objects may change during movement. So, each two points in space may not be linear geodesic and need not to be. Therefore, new metric is needed for non-linear distances like spherical surface. Then, a method is required for converting nonlinear distance to linear distance. Weyl introduced a metric with a conformal transformation in 1918 [1, 2].

Sluka shown that the pseudosymmetry as well as the Weylpseudosymmetry and the holomorphically projective-pseudosymmetry of $(M, J, g)$ reduces to the semisymmetry [3]. Antoniou and Pronko suggested the Hamiltonian approach for fluid mechanics based on the dynamics formulated in terms of Lagrangian variables [4]. Gilkey and Nikčević determined the space of algebraic pseudo-Hermitian KählerWeyl curvature tensors and the space of para-Hermitian Kähler. Weyl curvature tensors in dimension 4 and show that every algebraic possibility is geometrically realizable [5]. Gilkey and Nikčević gave an elementary proof of the fact that any 4-dimensional para-Hermitian manifold admits a unique para-Kähler-Weyl structure [6]. BrozosVázquez et al. shown that any algebraic possibility $\Sigma$ in this representation space can in fact be geometrically realized by a leftinvariant Kähler-Weyl structure on a 4-dimensional Lie group in either the Hermitian or the para-Hermitian setting [7]. Jelonek gave a description of compact conformally Kähler Einstein-Weyl manifolds whose Ricci tensor is Hermitian [8]. Kasap and Tekkoyun submitted 
mechanical systems on almost para/pseudo-Kähler-Weyl manifolds [9]. Kasap showed that Weyl-Euler-Lagrange and Weyl-Hamilton equations on $\mathbb{R}_{n}^{2 n}$ obtained a model of tangent manifolds of constant $W$-sectional curvature [10]. Kasap examined Weyl-Euler-Lagrange equations of motion on flat manifold [11].

\section{Preliminaries}

Definition 1. Let $M$ be a differentiable manifold of dimension $2 n$ and suppose $J$ is a differentiable vector bundle isomorphism $J: T M \rightarrow T M$ such that $J_{x}: T_{x} M \rightarrow T_{x} M$ is a (almost) complex structure for $T_{x} M$, i.e., $J^{2}=-I$, where $I$ is the identity (unit) operator on $V$. Then $J$ is called an almost-complex structure for the differentiable manifold $M$.

A manifold with a fixed (almost) complex structure is called an (almost) complex manifold.

Definition 2. A conformal map is a function which preserves angles. A conformal manifold is a differentiable manifold equipped with an equivalence class of (pseudo) Riemann metric tensors, in which two metrics $g^{\prime}$ and $g$ are equivalent if and only if

$$
g^{\prime}=\Psi^{2} g
$$

where $\Psi>0$ is a smooth positive function. An equivalence class of such metrics is known as a conformal metric or conformal class.

Definition 3. Two Riemann metrics $g_{1}$ and $g_{2}$ on $M$ are said to be conformally equivalent iff there exists a smooth function $f: M \rightarrow \mathbb{R}$ with

$$
e^{f} g_{1}=g_{2} \text {. }
$$

In this case, it is shown by $g_{1} \sim g_{2}$. 
Definition 4. Let $M$ be an $n$-dimensional smooth manifold. A pair $(M, C)$, a conformal structure on $M$ is an equivalence class $C$ of Riemann metrics on $M$, is called a conformal structure.

Theorem 1. Let $\nabla$ be a connection on $M$ and $g \in G$ be a fixed metric. $\nabla$ is compatible with $(M, G) \Leftrightarrow$ there exists a 1-form $\omega$ with $\nabla_{X} g+\omega(X) g=0[2]$.

Definition 5. A compatible torsion-free connection is called a Weyl connection. The triple $(M, G, \nabla)$ is a Weyl structure. A Weyl manifold is a conformal manifold equipped with a torsion free connection preserving the conformal structure, called a Weyl connection.

Theorem 2. To each metric $g \in G$ and 1-form $\omega$, there corresponds a unique Weyl connection $\nabla$ satisfying $\nabla_{X} g+\omega(X) g=0$. Here, $\nabla$ is given by the equation:

$$
\begin{aligned}
& g\left(\nabla_{X} Y, Z\right)=\frac{1}{2} \\
& \left\{\begin{array}{r}
X(g(Y, Z))+\omega(X) g(Y, Z)-g([X, Z], Y)+Y(g(X, Z))+\omega(Y) g(Z, X) \\
-g([Y, X], Z)-Z(g(X, Y))-\omega(Z) g(X, Y)-g([Z, Y], X)
\end{array}\right\} .
\end{aligned}
$$

Definition 6. Define a function $F:\{1$-forms on $M\} \times G \rightarrow\{$ Weyl connections by $F(g, \omega)=\nabla$, where $\nabla$ is the connection guaranteed by Theorem 2 . We say that $\nabla$ corresponds to $(g, \omega)$.

Proposition 1. F is surjective.

Proof. $F$ is surjective by Theorem 1. In fact, Theorem 1 shows that given a compatible, torsion-free connection $\nabla$, then for every $g \in G$, there exists a 1 -form $\omega$ with $F(g, \omega)=\nabla$. 
Proposition 2. $F(g, \omega)=F\left(e^{f} g, \eta\right)$ iff $\eta=\omega-d f$. So

$$
F\left(e^{f} g\right)=F(g)-d f
$$

where $G$ is a conformal structure. Note that a Riemann metric $g$ and a one-form $\omega$ determine a Weyl structure, namely, $F: G \rightarrow \wedge^{1} M$, where $G$ is the equivalence class of $g$ and $F\left(e^{f} g\right)=\omega-d f$ [12].

Proof. Suppose $F(g, \omega)=F\left(e^{f} g, \eta\right)=\nabla$. We have

$$
\begin{aligned}
0=\nabla_{X}\left(e^{f} g\right)+\eta(X) e^{f} g & =X\left(e^{f}\right) g+e^{f} \nabla_{X} g+\eta(X) e^{f} g \\
& =d f(X) e^{f} g+e^{f} \nabla_{X} g+\eta(X) e^{f} g .
\end{aligned}
$$

Therefore $\nabla_{X} g=-(d f(X)+\eta(X))$. On the other hand $\nabla_{X} g+\omega(X) g=0$ and $\omega=\eta+d f$. Conversely, suppose $\eta=\omega-d f$. Set $\nabla=F(g, \omega)$. To show $\nabla=F\left(e^{f} g, \eta\right)$, it suffices, by the uniqueness of Theorem 2 , to show $\nabla_{X}\left(e^{f} g\right)+\eta(X) e^{f} g=0$. Let us show the truth of this statement.

$$
\begin{aligned}
& \nabla_{X}\left(e^{f} g\right)=e^{f} d f(X) g+e^{f} \nabla_{X} g \text { and } \eta(X)=\omega(X)-d f(X) . \\
& \begin{aligned}
\nabla_{X}\left(e^{f} g\right)+\eta(X) e^{f} g & =e^{f} d f(X) g+e^{f} \nabla_{X} g+(\omega(X)-d f(X)) e^{f} g \\
& =e^{f} d f(X) g+e^{f} \nabla_{X} g+\omega(X) e^{f} g-d f(X) e^{f} g \\
& =e^{f}\left(\nabla_{X} g+\omega(X) g\right)=0 .
\end{aligned}
\end{aligned}
$$

Definition 7. Let $(M, g, J)$ be a Hermitian manifold of dimension $m$; here $J$ is an integrable complex structure on $M$ with $J g=g$. A Weyl structure on $(M, g)$ is a torsion free connection $\nabla$ such that $\nabla g=-2 \varnothing \otimes g$ for some smooth 1 -form $\varnothing$. This is a conformal theory; if $\widetilde{g}=e^{2 f} g$ is a conformally equivalent metric. We say that $(M, g, J, \nabla)$ is Kähler-Weyl if in addition $\nabla J=0$. Such structures are of interest only in dimension 4 as if $m \geqslant 6$, then $(M, g, J)$ is (locally) conformally equivalent to a Kähler manifold $(M, \tilde{g}, J)$ so that $\nabla$ is the Levi-Civita connection of $\widetilde{g}$. By 
contrast, in dimension 4, every Hermitian manifold $(M, g, J)$ admits a unique Kähler-Weyl structure where the associated 1-form $\varnothing$ is given by $\varnothing=-\frac{1}{2} d J \delta \Omega[5,13]$.

Definition 8. Let $(M, g)$ is conformally flat if for each point $x$ in $M$, there exists a neighbourhood $U$ of $x$ and a smooth function $f$ defined on $U$ such that $\left(U, e^{2 f} g\right)$ is flat. The function $f$ need not be defined on all of $M$ [14].

Weyl curvature tensor is a measure of the curvature of spacetime or a pseudo-Riemannian manifold. Like the Riemannian curvature tensor, the Weyl tensor expresses the tidal force that a object feels when moving along a geodesic. Weyl transformation is a local rescaling of the metric tensor:

$$
g_{a b}(x) \rightarrow e^{-2 \omega(x)} g_{a b}(x),
$$

which produces another metric in the same conformal class. A theory or an expression invariant under this transformation is called conformally invariant, or is said to possess Weyl symmetry. The Weyl symmetry is an important symmetry in conformal field theory.

\section{Kähler Manifold}

Definition 9. Let $M$ be a complex manifold with complex structure $J$ and compatible Riemannian metric $g=\langle\ldots\rangle$ as in $\langle J X, J Y\rangle=\langle X, Y\rangle$. $X=\sum_{i=1}^{n} X^{i} \frac{\partial}{\partial x_{i}}$ and $Y=\sum_{i=1}^{n} Y^{i} \frac{\partial}{\partial y_{i}}$ be two vector fields. The alternating 2 -form

$$
\omega(X, Y):=g(J X, Y),
$$

is called the associated Kähler form. We can retrieve $g$ from $\omega$,

$$
g(X, Y)=\omega(X, J Y) .
$$

We say that $g$ is a Kähler metric and that $M$ (together with $g$ ) is a Kähler manifold if $\omega$ is closed and $(M, g)$ is displayed in the form. 
Definition 10. Let $M$ be a complex manifold. A Riemannian metric on $M$ is called Hermitian if it is compatible with the complex structure $J$ of $M,\langle J X, J Y\rangle=\langle X, Y\rangle$. Then the associated differential two-form $\omega$ defined by

$$
\omega(X, Y)=\langle J X, Y>
$$

is called the Kähler form. It turns out that $\omega$ is closed if and only if $J$ is parallel. Then $M$ is called a Kähler manifold and the metric on $M$ a Kähler metric. Kähler manifolds are modelled on complex Euclidean space [15].

\section{Almost Pseudo/Para-Hermitian and Kähler-Weyl Geometry}

It is well-known that any Kähler Weyl curvature tensor is in fact Riemannian in dimension $n \geq 6$, this yields as a geometric consequence that any Kähler Weyl geometric structure is trivial for $n \geq 6$. On the contrary, the 4-dimensional setting is, as always, rather special as it turns out that there are Kähler Weyl curvature tensors which are not Riemannian if $n=4$. Since every Kähler Weyl curvature tensor is geometrically realizable and each side 4-dimensional Hermitian manifold admits a unique Kähler Weyl structure. There is also non-trivial 4-dimensional Hermitian Kähler Weyl manifold [6].

Definition 11. Let $n=2 \bar{n} \geq 4$. A triple $\left(M, g, J_{-}\right)$is said to be an almost pseudo-Hermitian manifold if $(M, g, \nabla)$ is with an almost complex structure $J_{-}$if $(M, g)$ is a pseudo-Riemannian manifold, if $J_{-}$ is an endomorphism of the tangent bundle so that $J_{-}^{2}=-i d$ and so that $J_{-} g=g ;\left(M, g, J_{-}\right)$is said to be a pseudo-Hermitian manifold with an integrable complex structure $J_{-}$if the Nijenhuis tensor

$$
N_{J_{-}}(x, y):=[x, y]+J_{-}\left[J_{-} x, y\right]+J_{-}\left[x, J_{-} y\right]-\left[J_{-} x, J_{-} y\right]
$$


vanishes or, equivalently, if there are local coordinates $\left(x^{1}, \ldots, x^{\tilde{m}}\right.$, $\left.y^{1}, \ldots, y^{\tilde{m}}\right)$ centered at an arbitrary point of $M$ so that

$$
J_{-} \partial_{x^{i}}=\partial_{y^{i}} \quad \text { and } \quad J_{-} \partial_{y^{i}}=-\partial_{x^{i}}, i=1, \ldots, n[16] .
$$

Let $\left(x^{i}, y^{i}\right)$ be a real coordinate system on a neighbourhood $U$ of any point $p$ of $M$, and $\left\{\frac{\partial}{\partial x^{i}}, \frac{\partial}{\partial y^{i}}\right\}$ and $\left\{d x^{i}, d y^{i}\right\}$ natural bases over $\mathbb{R}$ of the tangent space $T_{p}(M)$ and the cotangent space $T_{p}^{*}(M)$ of $M$, respectively. Thinking that $\left(M, g, J_{-}\right)$is a generalized Kählerian space form of dimension $2 n \geq 2$ and $J_{-}$-holomorphic sectional curvature; in which $g$ is the metric

$$
g=d x^{i} \otimes d x^{i}+d y^{i} \otimes-d y^{i},
$$

and $J_{-}$the almost product structure

$$
J_{-}=\frac{\partial}{\partial y^{i}} \otimes d x^{i}-\frac{\partial}{\partial x^{i}} \otimes d y^{i}
$$

As a result there is

$$
J_{-}\left(\frac{\partial}{\partial x^{i}}\right)=\frac{\partial}{\partial y^{i}}, J_{-}\left(\frac{\partial}{\partial y^{i}}\right)=-\frac{\partial}{\partial x^{i}} .
$$

The dual endomorphism $J_{-}^{*}$ of the cotangent space $T_{p}^{*}(M)$ at any point $p$ of manifold $M$ satisfies $J_{-}^{*}=-I d$ and is determined

$$
J_{-}^{*}\left(d x^{i}\right)=d y^{i}, J_{-}^{*}\left(d y^{i}\right)=-d x^{i} .
$$

A manifold with a Weyl structure is called a Weyl manifold. On every Weyl manifold, there is unique torsion-free compatible linear connection $\nabla$. Therefore, a Weyl manifold we denote by a triple $(M, g, \nabla)$. Assume that $n=2 \bar{n} \geq 4$. We say that $\left(M, g, J_{-}, \nabla\right)$ is an almost pseudo- 
Hermitian Weyl manifold if $(M, g, \nabla)$ is a Weyl manifold, if $J_{-}$is an almost complex structure on $T M$ (i.e., $J_{-}$is an endomorphism of $T M$ with $J_{-}^{2}=-i d$ and $\operatorname{Tr}\left(J_{-}\right)=0$ ), and if $J_{-}^{*} g=g$; necessarily $g$ has signature $(2 \bar{p}, 2 \bar{q})$ in this instance. It vanishes if and only if $J_{-}$is an integrable almost complex structure. If we extend by means of conformal structure ((16) and [17]), we can give equations as follows.

Proposition 3. Given any point $P \in M, f$ is a differentiable function, there exist local coordinates $\left(x_{1}, \ldots, x_{n}\right)$ centered at $P$ such that

$$
J_{-}\left(\frac{\partial}{\partial x^{i}}\right)=e^{2 f} \frac{\partial}{\partial y^{i}}, \quad J_{-}\left(\frac{\partial}{\partial y^{i}}\right)=-e^{-2 f} \frac{\partial}{\partial x^{i}}, \quad 1 \leq i \leq \bar{n} .
$$

Above, minus sign of the second structure must be selected for $J_{-}^{2}=-i d$.

Proof.

$$
\begin{aligned}
& J_{-}^{2}\left(\frac{\partial}{\partial x^{i}}\right)=J_{-} \circ J_{-}\left(\frac{\partial}{\partial x^{i}}\right)=J_{-}\left(e^{2 f} \frac{\partial}{\partial y^{i}}\right)=-e^{2 f} e^{-2 f} \frac{\partial}{\partial x^{i}}=-\frac{\partial}{\partial x^{i}}, \\
& J_{-}^{2}\left(\frac{\partial}{\partial y^{i}}\right)=J_{-} \circ J_{-}\left(\frac{\partial}{\partial y^{i}}\right)=-e^{-2 f} J_{-}\left(\frac{\partial}{\partial x^{i}}\right)=-e^{-2 f} e^{2 f} \frac{\partial}{\partial y^{i}}=-\frac{\partial}{\partial y^{i}} .
\end{aligned}
$$

Let $\left\{d x^{i}, d y^{i}\right\}$ be natural cobases over $\mathbb{R}$ of the cotangent space $T^{*} M$ of $M$, respectively. Taking into consideration $J_{-}$, then we can obtain the expressions about the dual $J_{-}^{*}$ as follows: defining by

$$
J_{-}^{*}\left(d x^{i}\right)=e^{2 f} d y^{i}, \quad J_{-}^{*}\left(d y^{i}\right)=-e^{-2 f} d x^{i}, \quad 1 \leq i \leq \bar{n} .
$$

Let $\left(M, g, J_{-}^{*}, \nabla\right)$ be an almost pseudo-Hermitian Weyl manifold. If $\nabla\left(J_{-}^{*}\right)=0$, then one says that this is a Kähler Weyl manifold. Note that necessarily $J_{-}^{*}$ is integrable in this setting. Also, similar to the above, structures are $J_{-}^{* 2}=-i d$. 
Theorem 3. Let $n \geq 6$. If $\left(M, g, J_{-}, \nabla\right)$ is a Kähler Weyl structure, then the associated Weyl structure is trivial, i.e., there is a conformal equivalent metric $\tilde{g}=e^{2 f} g$ so that $\left(M, g, J_{-}\right)$is Kähler and so that $\nabla=\nabla^{\tilde{g}}$, where exponential $(2 f)>0$ is a smooth positive exponential function. An equivalence class of such metrics is known as a conformal metric or conformal class [18].

\section{Hamilton Dynamics Equations}

Let $M$ is the base manifold and its cotangent manifold $T^{*} M$. By a symplectic form, we mean a 2 -form $\Phi$ on $T^{*} M$. Let $\left(T^{*} M, \Phi\right)$ be a symplectic manifold. A vector field $X_{H}: T^{*} M \rightarrow T T^{*} M$ is called Hamiltonian function if there is a $C^{1}$ function $H: T^{*} M \rightarrow \mathbb{R}$ such that Hamiltonian dynamical equation is determined by

$$
i_{X_{H}} \Phi=d H
$$

We say $X_{H}$ is locally Hamiltonian vector field. $\Phi$ is closed and also shows the canonical symplectic form so that $\Phi=-d \Omega, \Omega=J^{*}(\omega), J^{*}$ a dual of $J, \omega 1$-form on $T^{*} M$. The triple $\left(T^{*} M, \Phi, X_{H}\right)$ is named Hamiltonian system on the cotangent bundle $T^{*} M$. From the local expression for $X_{H}$, we see that $\left(q^{i}(t), p_{i}(t)\right)$ is an integral curve of $X_{H}$ iff Hamilton's equations is expressed as follow:

$$
q^{i}=\frac{\partial H}{\partial p_{i}}, \dot{p}_{i}=-\frac{\partial H}{\partial q^{i}}[19,20]
$$

\section{Conformal and Weyl-Hamiltonian Equations}

Here, we present Hamilton equations and Hamiltonian mechanical systems for quantum and classical mechanics constructed on the almost pseudo-Kähler Weyl manifolds. Let $\left(M, g, J_{-}^{*}\right)$ be the almost pseudo- 
Kähler Weyl manifolds. We will use the equality $g^{\prime}=e^{2 f} g$ for during this study ([17] and Theorem 3). Suppose that the complex structures, a Liouville form and a 1-form on the almost pseudo-Kähler Weyl manifolds are shown by $J_{-}^{*}, \Omega_{-}$, and $\omega$, respectively.

Proposition 4. Consider a 1-form such that

$$
\omega=\left(x^{i} d y^{i}+y^{i} d x^{i}\right)
$$

Then we obtain the Liouville form as follows:

$$
\Omega_{-}=J_{-}^{*}(\omega)=x^{i} e^{2 f} d y^{i}-y^{i} e^{-2 f} d x^{i} .
$$

It is well-known that if $\Phi_{-}$is a closed the almost pseudo-Kähler Weyl form on almost pseudo-Kähler Weyl manifolds $\left(M, g, J_{-}^{*}\right)$, then $\Phi_{-}$is also a symplectic structure on $\left(M, g, J_{-}^{*}\right)$. Therefore, the 2 -form $\Phi_{-}$ indicates the canonical symplectic form and derived from the 1 -form $\Omega_{-}$ to find to mechanical equations. Then the 2 -form is calculated as

$$
\begin{aligned}
& \Phi_{-}=-d\left(x^{i} e^{2 f} d y^{i}-y^{i} e^{-2 f} d x^{i}\right)
\end{aligned}
$$

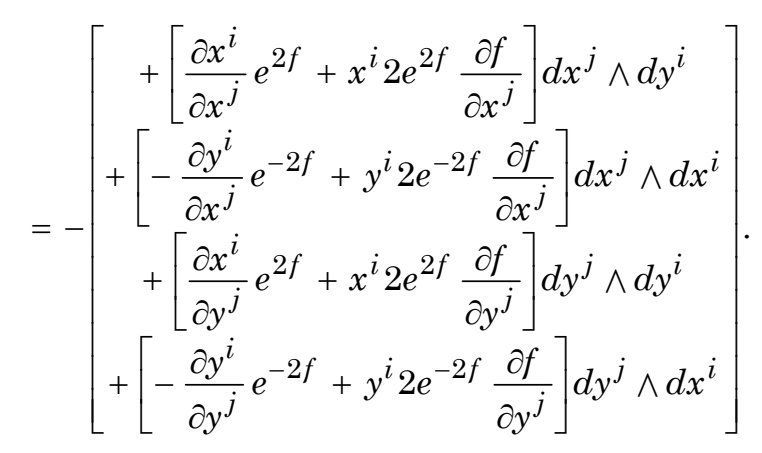

Take a vector field $Z_{H}$ so that called to be Hamiltonian vector field associated with Hamiltonian energy $H$ and determined by

$$
Z_{H}=X^{i} \frac{\partial}{\partial x^{i}}+Y^{i} \frac{\partial}{\partial y^{i}}
$$


So, we have

$$
\begin{aligned}
& i_{Z_{H}} \Phi_{-}=\Phi_{-}\left(Z_{H}\right)
\end{aligned}
$$

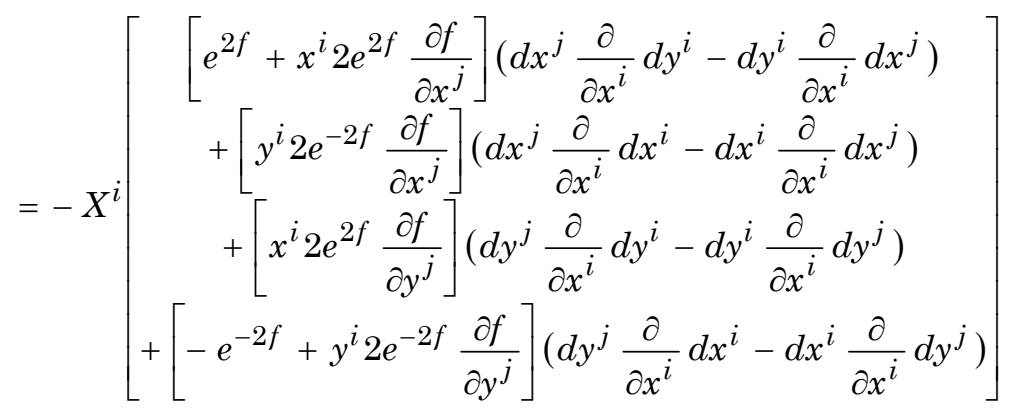

$$
\begin{aligned}
& -Y^{i}\left[\begin{array}{c}
{\left[e^{2 f}+x^{i} 2 e^{2 f} \frac{\partial f}{\partial x^{j}}\right]\left(d x^{j} \frac{\partial}{\partial y^{i}} d y^{i}-d y^{i} \frac{\partial}{\partial y^{i}} d x^{j}\right)} \\
+\left[y^{i} 2 e^{-2 f} \frac{\partial f}{\partial x^{j}}\right]\left(d x^{j} \frac{\partial}{\partial y^{i}} d x^{i}-d x^{i} \frac{\partial}{\partial y^{i}} d x^{j}\right) \\
+\left[x^{i} 2 e^{2 f} \frac{\partial f}{\partial y^{j}}\right]\left(d y^{j} \frac{\partial}{\partial y^{i}} d y^{i}-d y^{i} \frac{\partial}{\partial y^{i}} d y^{j}\right) \\
+\left[-e^{-2 f}+y^{i} 2 e^{-2 f} \frac{\partial f}{\partial y^{j}}\right]\left(d y^{j} \frac{\partial}{\partial y^{i}} d x^{i}-d x^{i} \frac{\partial}{\partial y^{i}} d y^{j}\right)
\end{array}\right],
\end{aligned}
$$

or

$$
\begin{aligned}
& \Phi\left(Z_{H}\right)=-X^{i}\left[\begin{array}{c}
{\left[e^{2 f}+x^{i} 2 e^{2 f} \frac{\partial f}{\partial x^{j}}\right] d y^{i}} \\
-\left[-e^{-2 f}+y^{i} 2 e^{-2 f} \frac{\partial f}{\partial y^{j}}\right] d y^{j}
\end{array}\right] \\
& -Y^{i}\left[\begin{array}{c}
-\left[e^{2 f}+x^{i} 2 e^{2 f} \frac{\partial f}{\partial x^{j}}\right] d x^{j} \\
\left.+\left[\begin{array}{c}
-2 f \\
-y^{i} 2 e^{-2 f} \frac{\partial f}{\partial y^{j}}
\end{array}\right] d x^{i}\right] .
\end{array}\right.
\end{aligned}
$$

Furthermore, the differential of Hamiltonian energy $H$ is obtained by

$$
d H=\frac{\partial H}{\partial x^{i}} d x^{i}+\frac{\partial H}{\partial y^{i}} d y^{i} .
$$


From $i_{Z_{H}} \Phi_{-}=d H$, the Hamiltonian vector field is found for $A=\left[e^{2 f}+x^{i} 2 e^{2 f} \frac{\partial f}{\partial x^{j}}+e^{-2 f}-y^{i} 2 e^{-2 f} \frac{\partial f}{\partial y^{j}}\right]$ as follows:

$$
Z_{H}=\frac{1}{A} \frac{\partial H}{\partial y^{i}} \frac{\partial}{\partial x^{i}}+\frac{-1}{A} \frac{\partial H}{\partial x^{i}} \frac{\partial}{\partial y^{i}}
$$

Consider the curve and its velocity vector $\alpha: I \subset \mathbb{R} \rightarrow M, \alpha(t)=\left(x^{i}, y^{i}\right)$ and

$$
\dot{\alpha}(t)=\frac{\partial \alpha}{\partial t}=\left(\frac{d x^{i}}{d t}, \frac{d y^{i}}{d t}\right),
$$

such that an integral curve of the Hamiltonian vector field $Z_{H}$, i.e.,

$$
Z_{H}(\alpha(t))=\frac{\partial \alpha}{\partial t}, \quad t \in I
$$

Then, we choose $i$ instead of $j$ and find the following equations:

$$
\begin{aligned}
& \frac{\partial H}{\partial y^{i}}=A \frac{d x^{i}}{d t} \\
& \frac{\partial H}{\partial x^{i}}=-A \frac{d y^{i}}{d t} .
\end{aligned}
$$

Hence, the equations introduced in (32) are named conformal Weyl Hamilton equations on the almost pseudo-Kähler Weyl manifolds and then the triple $\left(M, \Phi_{-}, Z_{H}\right)$ is said to be a Hamiltonian mechanical system on the almost pseudo-Kähler-Weyl manifolds.

\section{Weyl Hamiltonian for Conservative Mechanics Systems}

Now, we choose $F=i_{Z_{H}}$ and $g=\Phi_{-}$at (4) and by considering the Equation (20)

$$
i_{Z_{H}}\left(e^{2 f} \Phi_{-}\right)=i_{Z_{H}}\left(\Phi_{-}\right)-2 d f=\Phi_{-}\left(Z_{H}\right)-2 d f .
$$


The second part (20), according to the law of conservation of energy [20], will not change for conservative mechanics systems. So, we can write Weyl-Hamiltonian dynamic equation as follows:

$$
\Phi_{-}\left(Z_{H}\right)=d H+2 d f=d(H+2 f) .
$$

Using (32) and (34), we can obtain

$$
\begin{gathered}
\frac{\partial(H+2 f)}{\partial y^{i}}=A \frac{d x^{i}}{d t} \\
\frac{\partial(H+2 f)}{\partial x^{i}}=-A \frac{d y^{i}}{d t} .
\end{gathered}
$$

Hence, the equations introduced in (35) are named Weyl-Hamilton equations on the almost pseudo-Kähler Weyl manifolds $\left(M, \Phi_{-}, Z_{H}, F\right)$ for conservative mechanics systems. After that the triple $\left(M, \Phi_{-}, Z_{H}\right)$ is said to be a Weyl-Hamiltonian mechanical system on $\left(M, g, \nabla, J_{-}^{*}, F\right)$.

\section{Solution of Equations}

An electromagnetic field is a physical field produced by electrically charged objects. How the movement of objects in electrical, magnetically and gravitational fields force is very important. For instance, on a weather map, the surface wind velocity is defined by assigning a vector to each point on a map. So, each vector represents the speed and direction of the movement of air at that point.

The location of each object in space represented by three dimensions in physical space. Obtained in the above (32) system are partial differential equation and there are three independent variables. We can solve these equations by using the Maple computer program. First, implicit function at (32) will be selected as a special and we choose as special case of $f(x, y, t)=0, x(t)=\sin (t)$ and $y(t)=\cos (t)$. After, the 
figure of the Equation (32) has been drawn for the route of the movement of objects in the electromagnetic field. So, it is

$$
H(x, y, t)=\left(2 * y-2 * F_{1}(t)\right) * \cos (t)+2 * x * \sin (t),
$$

and graphics (36) is as follows for $F_{1}(t)=t$.

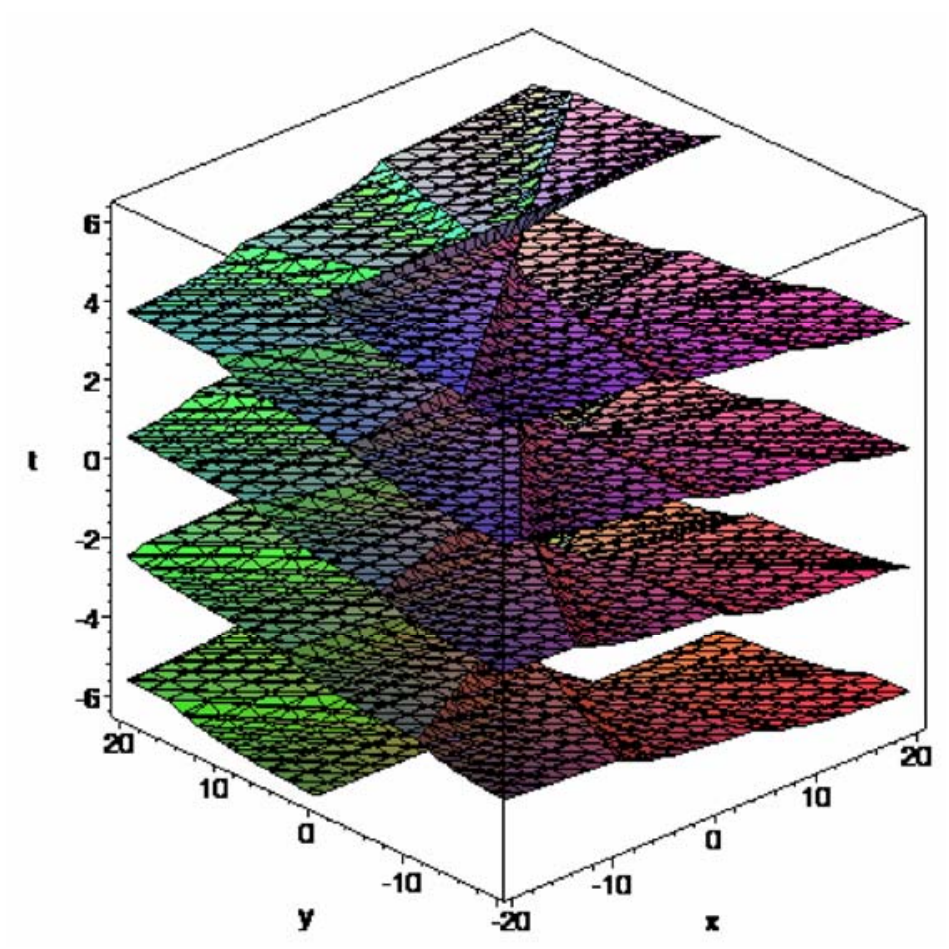

\section{Conclusion}

A classical field theory explain the study of how one or more physical fields interact with matter which is used in quantum and analytical mechanics.

In this study, the Hamilton mechanical equations (32), (35) derived on the almost pseudo-Kähler-Weyl manifolds. It may be suggested to deal with problems in electrical, magnetically and gravitational fields force for the path of movement (37) of defined space moving objects [21, 22]. 


\section{References}

[1] G. B. Folland, Weyl manifolds, J. Differential Geometry 4 (1970), 145-153.

[2] L. Kadosh, Topics in Weyl Geometry, Dissertationial, University of California, 1996.

[3] K. Sluka, Properties of the Weyl conformal curvature of Kähler-Norden manifolds, Steps in Differential Geometry, Proceedings of the Colloquium on Differential Geometry (2000), 25-30.

[4] I. Antoniou and G. P. Pronko, On the Hamiltonian description of fluid mechanics, (2001), 1-24.

[5] P. Gilkey and S. Nikčević, Kähler-Weyl manifolds of dimension 4, http://arxiv.org/pdf/1109.4532.pdf, (2011), 1-10.

[6] P. Gilkey and S. Nikčević, 4-dimensional (para)-Kähler-Weyl structures, http://arxiv.org/abs/1210.6769, (2012), 1-8.

[7] M. Brozos-Vázquez, E. Garia-Rio, P. Gilkey and R. Vázquez-Lorenzo, Homogeneous 4-dimensional Kähler-Weyl structures, Results. Math. 64 (2013), 357-369.

[8] W. Jelonek, Compact conformally Kähler Einstein-Weyl manifolds, Ann. Glob. Anal. Geom. 43 (2013), 19-29.

[9] Z. Kasap and M. Tekkoyun, Mechanical systems on almost para/pseudo-Kähler-Weyl manifolds, IJGMMP 10(5) (2013), 1-8.

[10] Z. Kasap, Weyl-mechanical systems on tangent manifolds of constant $W$-sectional curvature, IJGMMP 10(10) (2013), 1-13.

[11] Z. Kasap, Weyl-Euler-Lagrange equations of motion on flat manifold, Advances in Mathematical Physics (2015), 1-11.

[12] H. Weyl, Space-Time-Mattee, Dover Publ., 1922.

[13] P. Gilkey, S. Nikcević and U. Simon, Geometric realizations, curvature decompositions and Weyl manifolds, J. Geom. Phys. 61 (2011), 270-275.

[14] http://en.wikipedia.org/wiki/Conformal_class.

[15] W. Ballmann, Lectures on Kähler Manifolds, ESI Lectures in Mathematics and Physics, 2006.

[16] P. Gilkey and S. Nikčević, Kähler and para-Kähler, curvature Weyl manifolds, arXiv:1011.4844v1, (2010).

[17] R. Miron, D. Hrimiuc, H. Shimada and S. V. Sabau, The Geometry of Hamilton and Lagrange Spaces, FTPH 118, Kluwer Acad. Publ., 2001.

[18] P. Gilkey and S. Nikčević, Kähler and para-Kähler curvature Weyl manifolds, arXiv:1011.4844v1. 2010. 
[19] J. Klein, Escapes variationnels et mécanique, Ann. Inst. Fourier, Grenoble 12 (1962), $1-124$.

[20] M. de Leon and P. R. Rodrigues, Methods of differential geometry in analytical mechanics, Elsevier Sc. Pub. Com. Inc., Amsterdam, (1989), 263-397.

[21] B. Thidé, Electromagnetic field theory, (2012).

[22] R. G. Martín, Electromagnetic Field Theory for Physicists and Engineers: Fundamentals and Applications, Asignatura: Electrodinámica, Físicas, Granada, 2007. 